\title{
Does Dark Matter admixed pulsar exist?
}

\author{
Sajahan Molla ${ }^{1, a}$, Bidisha Ghosh ${ }^{2, b}$, Mehedi Kalam ${ }^{2, c}$ \\ ${ }^{1}$ Department of Physics, New Alipore College, L Block, New Alipore, Kolkata - 700 053, India \\ ${ }^{2}$ Department of Physics, Aliah University, IIA/27, Action Area II, Newtown, Kolkata -700156, India
}

Received: date / Accepted: date

\begin{abstract}
In this paper, we have considered a twofluid model assuming that the pulsars are made of ordinary matter admixed with dark matter.Contribution of dark matter comes from the fitting of the rotation curves of the SPARC sample of galaxies 95 . For this we have investigated the dark matter based on the Singular Isothermal Sphere (SIS) dark matter density profile in the galactic halo region. Considering this twofluid model, we have studied the physical features of the pulsars present in different galaxy in details. Here, we compute the probable radii, compactness $(\mathrm{u})$ and surface red-shift $\left(Z_{s}\right)$ of the four pulsars namely : PSR J1748-2021B in NGC 6440B galaxy, PSR J1911-5958A in NGC 6752 galaxy, PSR B1802-07 in NGC 6539 galaxy and PSR J1750-37A in NGC 6441 galaxy.
\end{abstract}

Keywords Compact star - Dark matter - Mass function $\cdot$ Radius $\cdot$ Compactness $\cdot$ Red-shift

\section{Introduction}

It was noticed that the study of compact objects take much attention to the astro physicist during the last few decade due to their unique properties compare to an Earth-based experiments. Compact objects comprise excellent natural laboratories to study, test and constrain new physics and/or alternative theories of gravity under extreme conditions. Compact relativistic objects such as white dwarfs, neutron stars and black hole are the last destiny of the evolved stars [1. The stars become stable when the outward degeneracy pressure provided by the Fermi gas balances the inward gravitational force. In case of white dwarfs the Fermi gas

\footnotetext{
${ }^{a}$ e-mail: sajahan.phy@gmail.com

be-mail: bidishaghosh.physics@gmail.com

${ }^{\mathrm{c}}$ e-mail: kalam@associates.iucaa.in
}

consists of electrons while in neutron stars Fermi gas consists of neutrons. Normally, neutron stars are composed mostly by neutrons while a new object, called strange stars are made of strange quark matter (SQM) or its conversion ( $\mathrm{u}, \mathrm{d}, \mathrm{s}$ quarks) and they may be enclosed to the core of the neutron star 2, 3]. It is familiar that neutron stars are bounded by gravitational attraction where as strange stars are bounded by strong interactions as well as gravitational attractions. Therefore, strange stars become more gravitationally bound than neutron stars. Since a strange star is more stable compared to a regular neutron star, its formation could interpret the origin of the huge amount of energy released in superluminous supernovae [4. This type of supernovae event happens about one out of every 1000 supernovae explosions and it is more than 100 times brighter than common supernovae. In most cases, a strange star and a neutron star can be separated on the basis of their vanishing surface energy density $[3,5,6,7,8,8$. Since after the birth of a neutron star, within a few second, its temperature becomes less than the Fermi energy, hence, for a given equation of state the mass and radius of the neutron star depend only on central density. Although, it is very difficult to find it's mass and radius simultaneously. We suggest to see a review work of Lattimer \& Prakash 2007 [9] for a detail study. From the solutions of Tolman-Oppenheimer-Volkoff equations, we can theoretically enumerate the mass and radii of the spherically-symmetric compact stars. The mass and radius of a compact star can be measured by pulsar timing, thermal emission from cooling stars, surface explosions and gravity wave emissions through observations.It is well known that the properties of the compact objects like mass and radius, crucially depend on the equation of state, unfortunately, which is poorly known to us. Truly, the most challenging task is to fix the ex- 
act Equation of State (EoS) to describe the structure of a compact star 10,11, 12,13,14,15]. Though few compact star's masses have been decided (to some extend), which are in binaries [16, 17, 18, 19,20 but there is no information about the radius. Therefore, the theoretical study of the stellar structure is required to support the correct direction for the newly observed stelar masses. In these ground some of the researcher's work on compact stars has been mentioned here [21,22, 23, 24, 25, 26, 27, 28, 29, 30, 31, 32, 33, 89, 35, 36, 37, 38, 39, 40.

In 1933 Zwicky discovered the dark matter while studying the dynamic properties of the Coma galaxy cluster [41. Whereas Rubin and Ford arrived at the similar conclusions about the existence of the dark matter with optical studies of galaxies like M31 [42] after few decades. We suggest to see the Ref. [43,44, a review work on dark matter. In fact, we are still unaware of the origin and nature of the dark matter. In reality, the kind of elementary particles playing the role of dark matter in the universe is one of the recent challenges of particle physics and modern cosmology. After an intensive study, cosmologists and particle physicists recently proposed many dark matter candidates to explain or constrain the properties [45, 46, 47, 48, 49, 50, 51,52, 53].

Though dark matter does't interact directly with normal matter, it can have significant gravitational effects on stellar objects [54,55,56]. It was reported that [57, 58, 59,60,61 the fermionic dark matter can have more gravitational effects on strange star's physical properties. Till today the spin of the dark matter particles remain unknown to us, one can think of the bosonic dark matter scenario. The authors Panotopoulos and Lopes 62] studied the effects of bosonic condensed dark matter on strange stars as far as the radial oscillations are concerned. It is generally accepted that dark matter particles are collisionless. Spergel and Steinhardt 63. inaugurated the idea that dark matter may have self-interaction in order to wipe out some apparent conflicts among the collisionless cold dark matter example and astrophysical observations. The dark matter core inside the neutron star considered as continuity in Ref. 64, whereas in Ref. 65, it was considered that dark matter halo enveloping the star. It was reported that in the presence of dark matter core scenario, possible effects on the maximum mass of a neutron star, its radius for any fixed mass, and its tidal deformability $\Lambda$ are in general reduced [61,66. Whereas an increase in tidal deformability $\Lambda$ was found in the dark matter halo model scenario 65 .

Generally Pulsar is a subclass of Neutron stars and it became an interesting object to the astrophysicists for the last few years. Freire et al. 67. measured the mass of the pulsar "PSR J1748-2021B" in NGC 6440B by using the Green Bank Telescope's S-band receiver and the Pulsar Spigot spectrometer and it comes out as $2.74 \pm 0.21 M_{\odot}$. In another work, Bassa et al. 68 present spectroscopic and photometric observations of the optical counterpart to PSR J1911-5958A, a millisecond pulsar located in the globular cluster NGC 6752 and it's mass comes out as $1.40_{-0.10}^{+0.16} M_{\odot}$. On the other hand, S.E. Thorsett and D. Chakrabarty 69] reported the measurement of the mass of the PSR B1802-07 which is in the globular cluster NGC 6539, as $1.26_{-0.17}^{+0.08} M_{\odot}$. Freire et al. 70] have also determined the mass of the pulsar "PSR J1750-37A" in NGC 6441 as $1.26_{-0.36}^{+0.39} M_{\odot}$.

The Singular Isothermal Sphere (SIS) model is very useful for gravitational lensing 71 . Also, so many observational evidences are there where they are consistent with SIS profiles.This motivates us to study the Singular Isothermal Sphere(SIS) model[71] to study the dark matter.

Also, we have been motivated by the previous articles on dark matter neutron star reported in well known journals $[55,58,61,72,73,74,75,76,77$. The motto of the present article is to study the existence of dark matter with ordinary matter in pulsars located in various galaxies, namely, PSR J1748-2021B in NGC 6440B, PSR J1911-5958A in NGC 6752, PSR B1802-07 in NGC 6539, PSR J1750-37A in NGC 6441. We have come to a conclusion that there is every possibility of existence of dark matter admixed with ordinary matter in the above mentioned pulsars.

\section{Interior Spacetime}

Consider a static and spherically symmetric star with interior spacetime as

$d s^{2}=-e^{\nu(r)} d t^{2}+e^{\lambda(r)} d r^{2}+r^{2}\left(d \theta^{2}+\sin ^{2} \theta d \phi^{2}\right)$

Inspired from many previous revealed famous articles $[78,79,80,81,82,83,84,85,86,87,88,89,90$, we have taken static, spherical symmetric metric for this pulsar model. The Einstein field equations for the metric Eq. (1) obtained as (taking $G=c=1$ )

$$
\begin{aligned}
8 \pi \rho & =e^{-\lambda}\left(\frac{\lambda^{\prime}}{r}-\frac{1}{r^{2}}\right)+\frac{1}{r^{2}} \\
8 \pi p_{r} & =e^{-\lambda}\left(\frac{\nu^{\prime}}{r}+\frac{1}{r^{2}}\right)-\frac{1}{r^{2}} \\
8 \pi p_{t} & =\frac{e^{-\lambda}}{2}\left(\frac{\left(\nu^{\prime}\right)^{2}-\lambda^{\prime} \nu^{\prime}}{2}+\frac{\nu^{\prime}-\lambda^{\prime}}{r}+\nu^{\prime \prime}\right)
\end{aligned}
$$

According to H. Heintzmann [91,

$e^{\nu}=A^{2}\left(1+a r^{2}\right)^{3}$ 
and

$e^{-\lambda}=\left[1-\frac{3 a r^{2}}{2}\left(\frac{1+C\left(1+4 a r^{2}\right)^{-\frac{1}{2}}}{1+a r^{2}}\right)\right]$

Where A (dimensionless), C (dimensionless) and a ( length $^{-2}$ ) are constants (using geometric units $\mathrm{G}=\mathrm{c}=1$ ). Therefore,

$\nu^{\prime}=\frac{6 a r}{1+a r^{2}}$

$\nu^{\prime \prime}=-\frac{6 a\left(-1+a r^{2}\right)}{\left(1+a r^{2}\right)^{2}}$

$\lambda^{\prime}=-\left[6 a r\left(C+2 a C r^{2}-2 a^{2} C r^{4}+\left(1+4 a r^{2}\right)^{\frac{3}{2}}\right)\right]$
$\times\left[\left(1+a r^{2}\right)\left(1+4 a r^{2}\right)\right.$
$\left.\left(-2 \sqrt{1+4 a r^{2}}+a r^{2}\left(3 C+\sqrt{1+4 a r^{2}}\right)\right)\right]^{-\frac{1}{2}}$

We also assume that the energy-momentum tensor for the matter distribution of the compact star has the standard form as

$T_{i j}=\operatorname{diag}\left(-\rho, p_{r}, p_{t}, p_{t}\right)$

where $\rho$ is the energy density, $p_{r}$ and $p_{t}$ are the radial and transverse pressure respectively.

The singular isothermal sphere (SIS) density profile is the simplest model of the matter distribution in an astrophysical system. F. Brimioulle et al. 92 have mentioned that, dark matter is the dominant part of the galaxy. They have done galaxy-galaxy lensing (GGL) study based on imaging data from the Canada-FranceHawaii Telescope Legacy Survey Wide. They have fitted three galactic halo profiles to the lensing signal, among the three, one is singular isothermal sphere (SIS) dark matter density profile.

Also, M. Oguri et al. 93. have considered dark halo density profile as the SIS as well as NFW density profile. This motivates us to take the dark matter density profile as SIS density profile.

SIS model for dark matter energy density which is applicable to the stellar cores with no nuclear burning i.e. for compact star is as follows: $\rho_{d}(r)=\frac{K}{2 \pi G r^{2}} \&$ $p_{d}(r)=\frac{m K}{2 \pi G r^{2}}$, where $\mathrm{K}, \mathrm{m}$ are constant 94 .

The tangential velocity of the halo region, $v_{\text {halo }}^{2}$ can be written as

$v_{\text {halo }}^{2}=\frac{G}{R} \int_{0}^{R} 4 \pi r^{2} \rho_{d}(r) d r=2 K$.

The tangential velocity at the halo region, $v_{\text {halo }}^{2}$ for dwarf galaxies are shown in Table 1 and for massive
Table 1 Circular velocity of the flat part of the rotation curves of galaxies $\left(v_{o b s}\right)$, rotational velocity of halos $\left(v_{\text {halo }}\right)$ and the $\mathrm{K}$ values of dwarf galaxies which is calculated from the galactic rotational curve fit of dwarf galaxies.

\begin{tabular}{lccc}
\hline Galaxy & $v_{o b s}^{2}\left(\times 10^{-07}\right)$ & $v_{\text {halo }}^{2}\left(\times 10^{-08}\right)$ & $K\left(\times 10^{-08}\right)$ \\
\hline NGC 3769 & 1.764 & 9.282 & 4.641 \\
NGC 3877 & 3.249 & 8.853 & 4.426 \\
NGC 3917 & 2.116 & 8.853 & 4.426 \\
NGC 3949 & 3.173 & 4.895 & 2.447 \\
NGC 3972 & 1.995 & 7.567 & 3.783 \\
NGC 4051 & 2.880 & 7.077 & 3.538 \\
NGC 4085 & 2.055 & 2.581 & 1.290 \\
NGC 4088 & 3.680 & 6.032 & 3.016 \\
NGC 4183 & 1.469 & 8.317 & 4.158 \\
NGC 4214 & 0.722 & 4.867 & 2.433 \\
NGC 4217 & 4.053 & 8.650 & 4.325 \\
NGC 7793 & 1.495 & 2.380 & 1.190 \\
NGC 0024 & 1.344 & 7.712 & 3.856 \\
NGC 0300 & 1.045 & 5.471 & 2.735 \\
UGC 00191 & 0.781 & 3.970 & 1.985 \\
UGC 00634 & 1.296 & 9.847 & 4.923 \\
UGC 00731 & 0.608 & 3.282 & 1.641 \\
UGC 00891 & 0.452 & 2.955 & 1.477 \\
UGC 01230 & 1.418 & 6.934 & 3.467 \\
UGC 05716 & 0.620 & 3.756 & 1.878 \\
UGC 05721 & 0.758 & 4.568 & 2.284 \\
UGCA 442 & 0.371 & 2.314 & 1.157 \\
DDO 161 & 0.506 & 2.849 & 1.424 \\
F563-V2 & 1.547 & 3.907 & 1.953 \\
F565-V2 & 0.767 & 4.411 & 2.205 \\
\hline
\end{tabular}

spiral galaxies are shown in Table 2. It was calculated from the fitting of the rotation curves of the SPARC sample of galaxies 95]. $v_{\text {halo }}^{2}$ has been calculated from the equation given below :

$v_{\text {halo }}^{2}=v_{\text {obs }}^{2}-\left(v_{\text {star }}^{2}+v_{\text {gas }}^{2}\right) ;$

It is to be mentioned here that the $\mathrm{K}$ values for both dwarf and spiral galaxies are of the order of $10^{-7}$ to $10^{-8}$ and that value of $\mathrm{K}$ comes from $v_{\text {halo }}^{2}$, the observational data of galactic rotational curve. Moreover, this $\mathrm{K}$ has an important role to the density distribution of the dark matter haloes.

Now, we consider the pulsars are made of ordinary matter admixed with condensed dark matter. Therefore, effective density and pressure can be written as

$\rho_{e f f}=\rho+\rho_{d}$

$p_{\text {eff }}=p-p_{d}$

The presence of dark matter in addition with normal matter the Einstein field equations for the metric Eq. (1) can be obtained as (taking $G=c=1$ )

$$
\begin{aligned}
\rho & =\frac{1}{8 \pi}\left[e^{-\lambda}\left(\frac{\lambda^{\prime}}{r}-\frac{1}{r^{2}}\right)+\frac{1}{r^{2}}\right]-\frac{K}{2 \pi r^{2}} \\
p_{r} & =\frac{1}{8 \pi}\left[e^{-\lambda}\left(\frac{\nu^{\prime}}{r}+\frac{1}{r^{2}}\right)-\frac{1}{r^{2}}\right]+\frac{m K}{2 \pi r^{2}}
\end{aligned}
$$


Table 2 Circular velocity of the flat part of the rotation curves of galaxies $\left(v_{o b s}\right)$, rotational velocity of halos ( $\left.v_{\text {halo }}\right)$ and the $\mathrm{K}$ values of spiral galaxies, which is calculated from the galactic rotational curve fit of spiral galaxies.

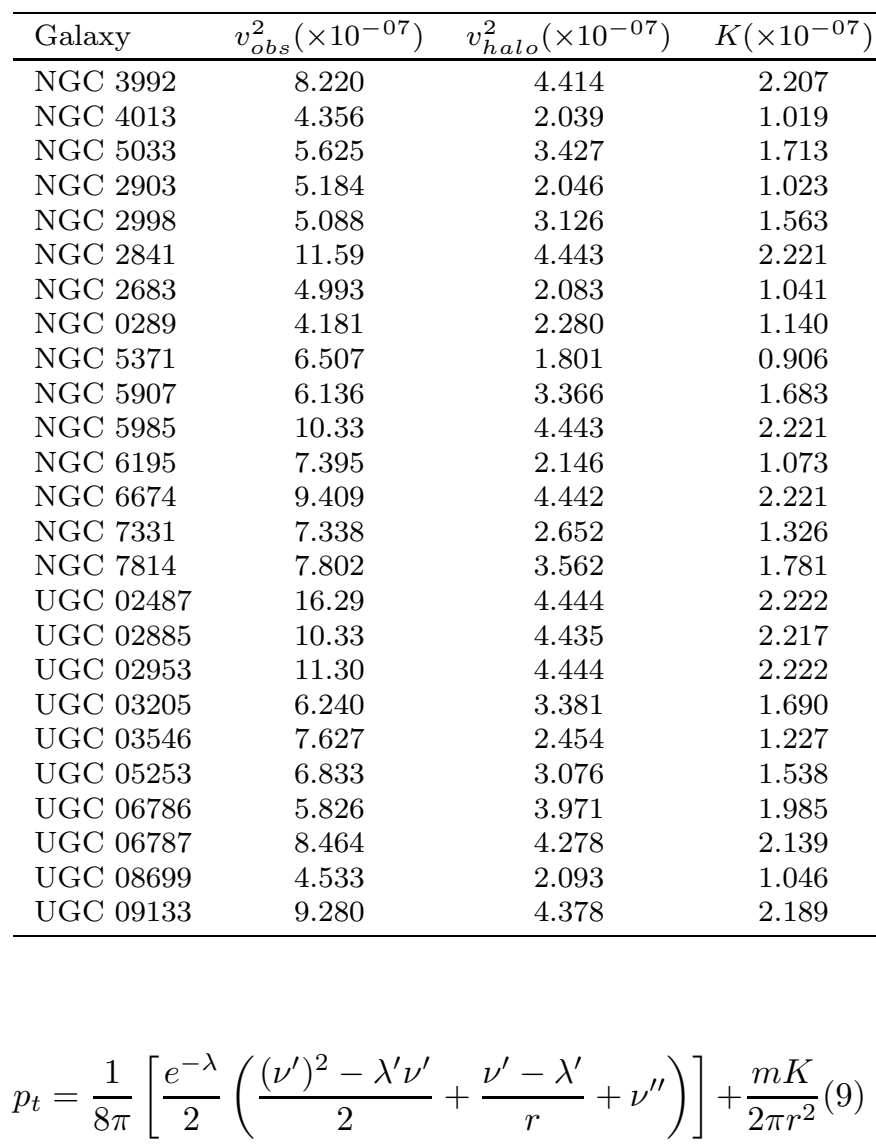

Fig. 1 shows that, the matter density and pressure both are maximum at the centre and decreases monotonically towards the boundary. Thus, the energy density and the pressure are well behaved in the interior of the stellar structure. The anisotropic parameter $\Delta(r)=$ $\left(p_{t}-p_{r}\right)$ representing the anisotropic stress is given by Fig. 1. The 'anisotropy' will be directed outward when $p_{t}>p_{r}$ i.e. $\Delta>0$, and inward when $p_{t}<p_{r}$ i.e. $\Delta<0$. It is apparent from the Fig. 1 of our model that a repulsive 'anisotropic' force $(\Delta>0)$ allows the construction of more massive distributions. It is to be mentioned here that, we set the values of the constants $K=10^{-8}$ ( as observed $K$ varies from $10^{-7}$ to $10^{-8}$ ), $a=0.003 \mathrm{~km}^{-2}$, $C=0.8$ and $m=10^{-6}$, such that the pressure drops to zero at the boundary.

\section{Exploration of Physical properties}

In this section we will investigate the following physical features of the compact object under these model:

\subsection{Energy conditions}

From Fig. 2 we observe that all the energy conditions namely, null energy condition(NEC), weak energy condition(WEC), strong energy condition(SEC) and dominant energy condition(DEC) are satisfied in our stellar model.

(i) NEC: $p+\rho \geq 0$,

(ii) WEC: $p+\rho \geq 0, \quad \rho \geq 0$,

(iii) SEC: $p+\rho \geq 0, \quad 3 p+\rho \geq 0$,

(iv) DEC: $\rho>|p|$.

\subsection{TOV equation}

For an anisotropic fluid distribution, the generalized TOV equation has the form

$\frac{d p_{r}}{d r}+\frac{1}{2} \nu^{\prime}\left(\rho+p_{r}\right)+\frac{2}{r}\left(p_{r}-p_{t}\right)=0$.

Following Ref. 96], we write the above equation as

$-\frac{M_{G}\left(\rho+p_{r}\right)}{r^{2}} e^{\frac{\lambda-\nu}{2}}-\frac{d p_{r}}{d r}+\frac{2}{r}\left(p_{t}-p_{r}\right)=0$,

Where $M_{G}(r)$ is the gravitational mass inside a sphere of radius $r$ and is given by

$M_{G}(r)=\frac{1}{2} r^{2} e^{\frac{\nu-\lambda}{2}} \nu^{\prime}$.

which can be derived from the Tolman-Whittaker formula and the Einstein's field equations. The modified TOV equation describes the equilibrium condition for the compact star subject to gravitational $\left(F_{g}\right)$ and hydrostatic $\left(F_{h}\right)$ plus another force due to the anisotropic $\left(F_{a}\right)$ nature of the stellar object as

$F_{g}+F_{h}+F_{a}=0$,

Where,

$F_{g}=-\frac{1}{2} \nu^{\prime}\left(\rho+p_{r}\right)$

$F_{h}=-\frac{d p_{r}}{d r}$

$F_{a}=\frac{2}{r}\left(p_{t}-p_{r}\right)$

We plot (Fig. 3) the behavior of pressure anisotropy, gravitational and hydrostatic forces in the stellar interior, which clearly shows that the static equilibrium configurations do exist due to the combined effect of pressure anisotropy, gravitational and hydrostatic forces.

\subsection{Stability}

For a physically acceptable stellar model, one expects that the speed of sound should be within the range $0 \leq$ 

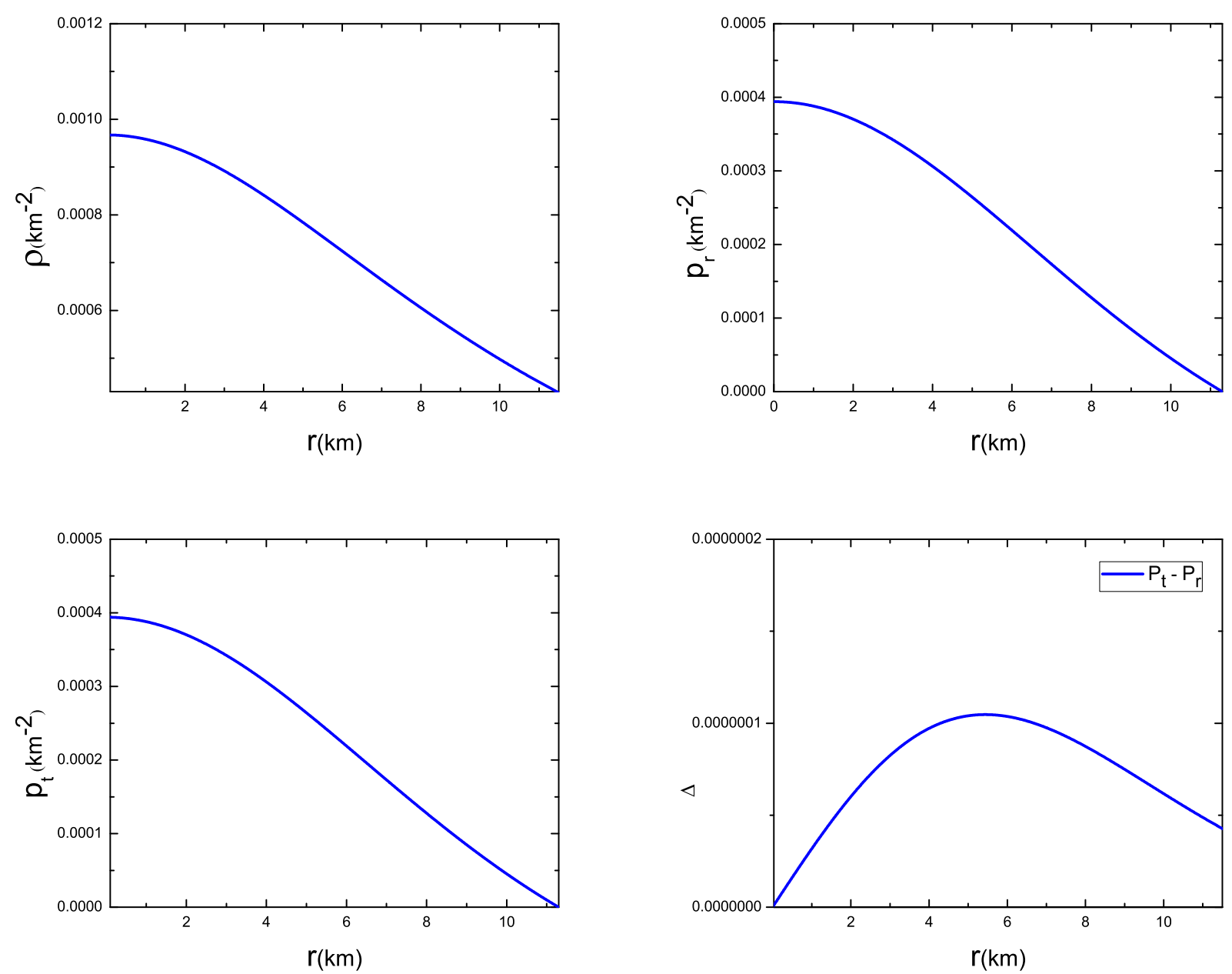

Fig. 1 Matter density $(\rho)-\operatorname{radius}(\mathrm{r})$ and $\operatorname{pressure}(p)$ - radius(r) variation at the pulsar interior.

$v_{s}^{2}=\left(\frac{d p}{d \rho}\right) \leq 1[97,98$. In our model, we plot the radial and transverse sound speeds in Fig. 4 and observed that these parameters satisfies the inequalities $0 \leq v_{s r}^{2} \leq 1$ and $0 \leq v_{s t}^{2} \leq 1$ everywhere within the stellar object. Since, $0 \leq v_{s r}^{2} \leq 1$ and $0 \leq v_{s t}^{2} \leq 1$, therefore, $\mid v_{s t}^{2}-$ $v_{s r}^{2} \mid \leq 1$. In Fig. 4, we have plotted $\left|v_{s t}^{2}-v_{s r}^{2}\right|$ for such verification. These results shows that our dark matter admixed pulsars model is stable.

In our stellar model, the adiabatic $\operatorname{index}(\gamma)$ satisfies the inequality $\gamma=\frac{\rho+p_{r}}{p_{r}} \frac{d p_{r}}{d \rho}>\frac{4}{3}$ everywhere within the stellar interior (Fig. 4) which also verifies the dynamical stability of our stellar model in presence of thermal radiation. This type of stability executed by several author like Chandrasekhar 99], Bardeen et al. [100, Knutsen 101, Mak and Harko [102] gradually in their work.

\subsection{Matching Conditions}

The interior metric of the stellar body should match with the Schwarzschild exterior metric at the boundary $(r=b)$.

$d s^{2}=-\left(1-\frac{2 M}{r}\right) d t^{2}+\left(1-\frac{2 M}{r}\right)^{-1} d r^{2}+r^{2} d \Omega^{2}(17)$

Assuming the continuity of the metric functions $g_{t t}, g_{r r}$ and $\frac{\partial g_{t t}}{\partial r}$ at the boundary, we get Continuity of the metric function across the boundary yields the compactification factor as

$\frac{M}{b}=\frac{1}{2}\left[\frac{3 a b^{2}\left(1+C\left(1+4 a b^{2}\right)^{-\frac{1}{2}}\right)}{2\left(1+a b^{2}\right)}\right]$

\subsection{Mass-Radius relation and Surface redshift}

According to Buchdahl [103], maximum allowable massradius ratio for a static spherically symmetric perfect 


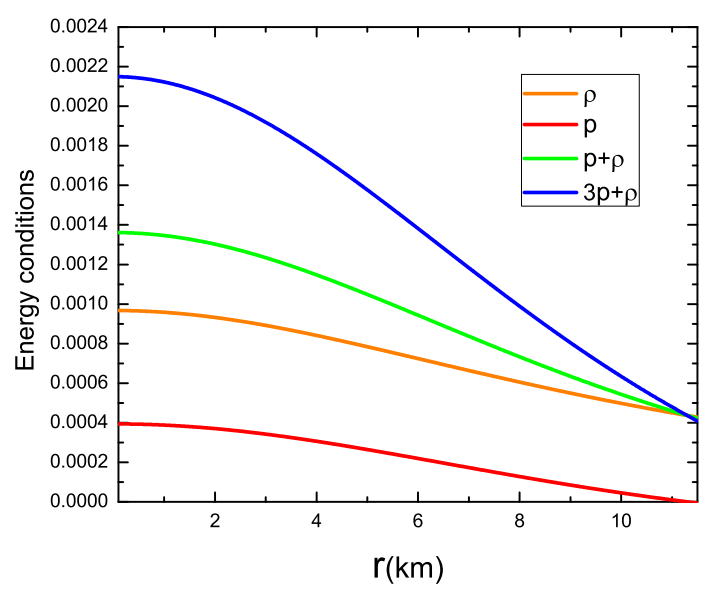

Fig. 2 Energy conditions variation at the pulsar interior.

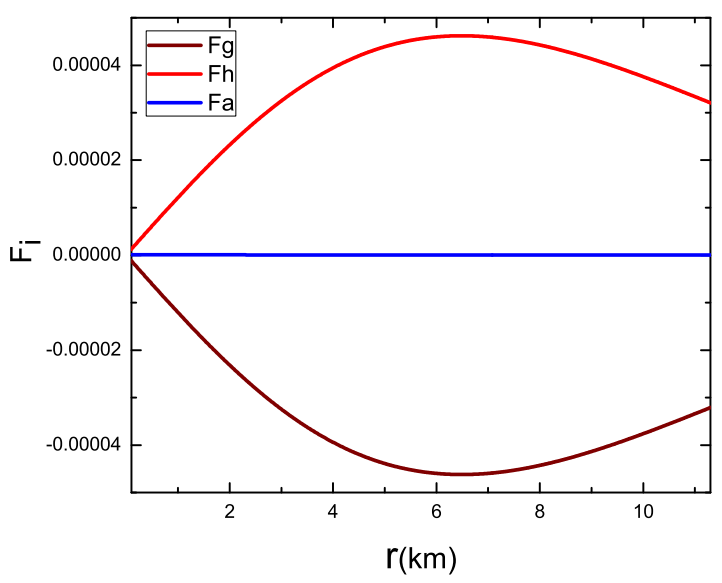

Fig. 3 Behaviors of pressure anisotropy $\left(F_{a}\right)$, gravitational $\left(F_{g}\right)$ and hydrostatic $\left(F_{h}\right)$ forces at the pulsar interior.

fluid sphere should be $\frac{\text { Mass }}{\text { Radius }}<\frac{4}{9}$. We have calculated the gravitational mass $(\mathrm{M})$ as

$M=4 \pi \int_{0}^{b} \rho_{\text {eff }} r^{2} d r=\frac{3 a b^{3}\left(1+C\left(1+4 a b^{2}\right)^{-\frac{1}{2}}\right)}{4\left(1+a b^{2}\right)}$

where $b$ is the radius of the pulsar. Therefore, the compactness $(u)$ of the pulsar can be written as

$u=\frac{M}{b}=\frac{1}{2}\left[\frac{3 a b^{2}\left(1+C\left(1+4 a b^{2}\right)^{-\frac{1}{2}}\right)}{2\left(1+a b^{2}\right)}\right]$

The variation of mass function and compactness of the pulsar are shown in Fig. 5 and Fig. 6 respectively. The surface redshift $\left(Z_{s}\right)$ corresponding to the above compactness $(u)$ can be written as

$$
Z_{s}=[1-2 u]^{-\frac{1}{2}}-1
$$

$$
Z_{s}=-1+\frac{1}{\sqrt{1-\frac{3 a b^{2}\left(1+\frac{C}{\sqrt{1+4 a b^{2}}}\right)}{2+2 a b^{2}}}}
$$

Therefore from Fig. 7, the maximum surface redshift for the different pulsars can be obtained easily. The radii, compactness and surface redshift of the different pulsars are evaluated from Fig. 8, Eq. (18) and Eq. (20) and a comparative analysis has been done in Table 1.

\section{Discussion and Concluding Remarks}

In the present work, we investigate the nature of the pulsars present in different galaxy by taking the Heintzmann IIa metric. In general pulsars, due to their high density, becomes anisotropic in nature. That's why we consider the anisotropic behaviour of the pulsar to make more generalized model.Also, we have been motivated by the previous articles on dark matter neutron star reported in well known journals $[55,58,61,72,73,74,75$, 76. 77. But our approach is quite different from these articles. Conventionally, the mass-radius curve of compact stars are calculated under a given equation of state for various values of central density; by a given value of the central density, the mass and radius of a compact star are fixed. According to our model, different pulsars are depends on the same parameter values $K=$ $10^{-8}$ ( as observed $K$ varies from $10^{-7}$ to $10^{-8}$ ), $a=$ $0.003 \mathrm{~km}^{-2}, C=0.8$ and $m=10^{-6}$, such that it obeys the standard star model condition ( i.e. pressure drops to zero at the boundary etc.). Consequently, they have the same central density and the same equation of state. Therefore, interestingly, if we starts from the centre with a certain central density, the model of a compact star can be determined by stopping at any radius where pressure becomes zero. We think this model will give new dimension to study of compact stars.

As the structure of the pulsar are still not known,we have considered a two-fluid model assuming that the pulsars are made of ordinary matter admixed with dark matter having a characteristic parameter $\rho_{d}$. Contribution of dark matter comes from the fitting of the rotation curves of the SPARC sample of galaxies 95. For this we have investigated the dark matter based on the Singular Isothermal Sphere (SIS) dark matter density profile in the galactic halo region.

Main motivation of the present article is to study the pulsars ( consists of dark matter with ordinary matter) presents in different galaxies, namely, PSR J17482021B in NGC 6440B, PSR J1911-5958A in NGC 6752, PSR B1802-07 in NGC 6539, PSR J1750-37A in NGC 

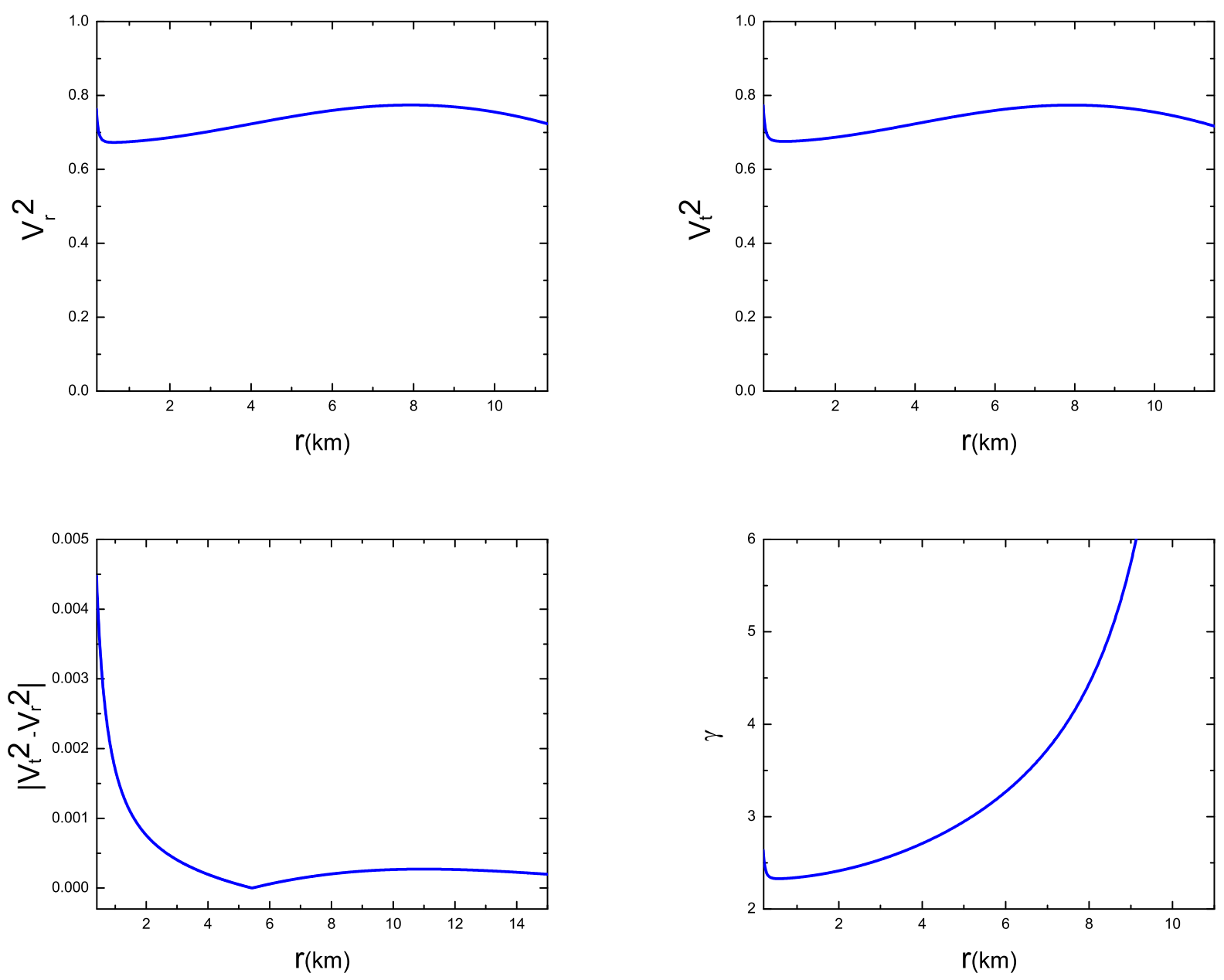

Fig. 4 Sound speed $\left(v^{2}\right)$ - radius $(r)$ and adiabatic index $(\gamma)$ - radius $(r)$ variation at the pulsar interior.

Table 3 Evaluated parameters for pulsars

\begin{tabular}{lllll}
\hline Star & Observed Mass $\left(M_{\odot}\right)$ & Radius from Model(in km) & Compactness from Model & Redshift from Model \\
\hline PSR J1748-2021B & $2.74 \pm 0.21$ & $12.005 \pm 0.425$ & $0.336 \pm 0.014$ & $0.750 \pm 0.075$ \\
PSR J1911-5958A & $1.40_{-0.10}^{+0.16}$ & $8.72 \leq \mathrm{R} \leq 9.4$ & $0.220 \leq \mathrm{u} \leq 0.245$ & $0.336 \leq Z_{s} \leq 0.340$ \\
PSR J1750-37A & $1.26_{-0.36}^{+0.39}$ & $7.53 \leq \mathrm{R} \leq 9.62$ & $0.176 \leq \mathrm{u} \leq 0.253$ & $0.243 \leq Z_{s} \leq 0.422$ \\
PSR B1802-07 & $1.26_{-0.17}^{+0.08}$ & $8.13 \leq \mathrm{R} \leq 8.83$ & $0.198 \leq \mathrm{u} \leq 0.224$ & $0.287 \leq Z_{s} \leq 0.346$ \\
\hline
\end{tabular}

6441.

Successfully, we find an analytical solution to the fluid sphere which are quite interesting in connection to several physical features, which are as follows:

1. In our model, density and pressure at the interior of the pulsar are well behaved (Fig. 1). Pressure and density are both maximum at the centre and monotonically decreasing towards the boundary. Here, we assume the values of constants $(a, C)$ in the metric and $\mathrm{m}, K=10^{-8}$ ( as observed $K$ varies from $10^{-7}$ to $10^{-8}$ ) in such a way that pressure must vanish at the boundary. Our pulsar model satisfies all the energy conditions, TOV equation and Herrera's stability condition [97]. It is also stable with respect to infinitesimal radial thermal perturbations. From the mass function (Eq. 17), all desired interior features of a pulsar can be evaluated which satisfies Buchdahl mass-radius relation $\left(\frac{2 M}{R}<\frac{8}{9}\right)$ (Figs. 5 and 6$)$. The surface redshift of the pulsar are found within the standard value $\left(Z_{s} \leq 0.85\right)$ which is satisfactory (Fig. 7) [104. 


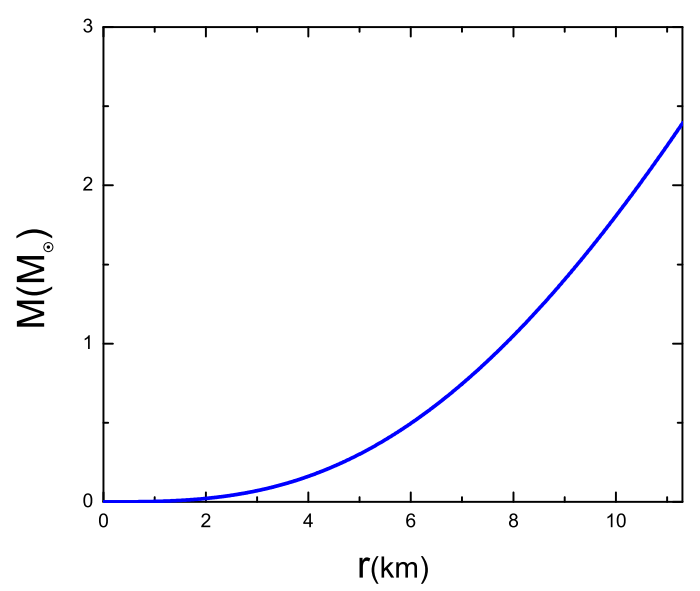

Fig. 5 Mass function $\mathrm{M}(\mathrm{r})$ variation at the pulsar interior.

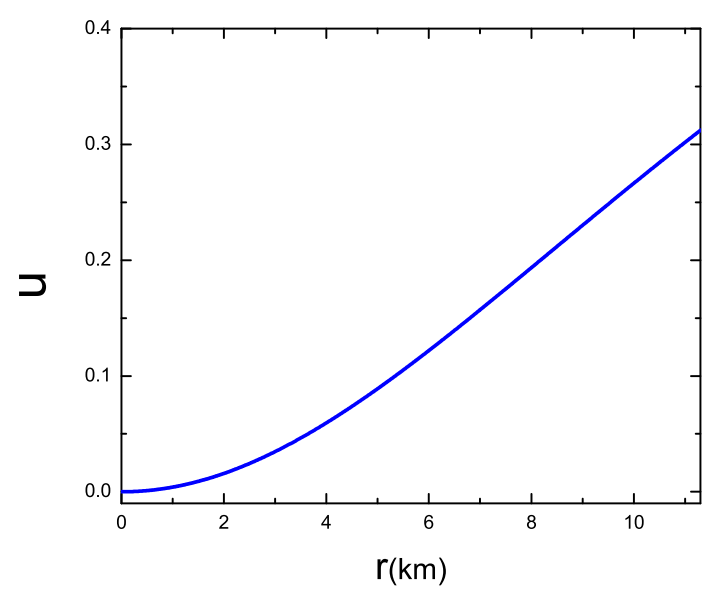

Fig. 6 Variation of the compactness (u) at the pulsar interior.

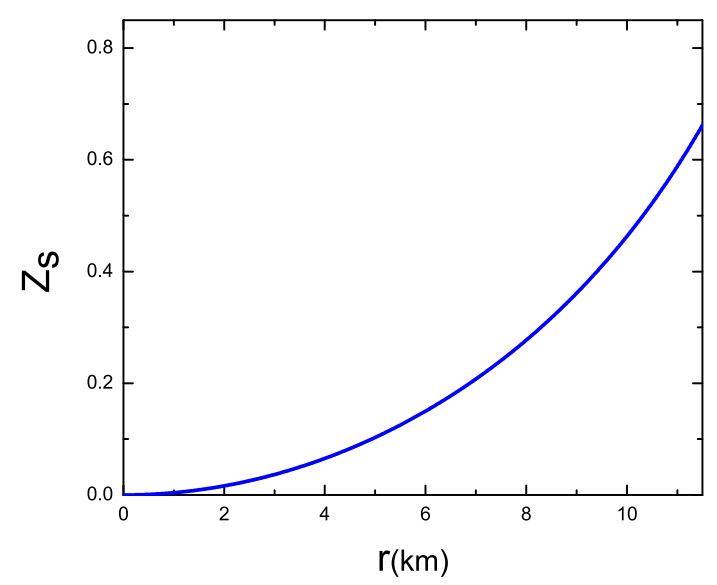

Fig. 7 Variation of the surface redshift $\left(Z_{s}\right)$ at the pulsar interior.
2. From our mass function graphs fig. 8, Eq. (18) and Eq. (20), we obtain the radii, compactness and surface red-shift of four pulsars in the various galaxy namely: PSR J1748-2021B in NGC 6440B, PSR J19115958A in NGC 6752, PSR B1802-07 in NGC 6539 and PSR J1750-37A in NGC 6441. The detail comparison chart are shown in Table 3.

It is to be mentioned here that the $K$ values for both dwarf and spiral galaxies are of the order of $10^{-7}$ to $10^{-8}$ and that value of $\mathrm{K}$ comes from $v_{\text {halo }}^{2}$, the observational data of galactic rotational curve. Moreover, this $\mathrm{K}$ has an important role to the density distribution of the dark matter haloes. In our calculation, we consider the value of $\mathrm{K}$ as $10^{-8}$ (i.e. contribution comes from Galactic Rotation Curve data).

In conclusion, we can say that incorporation of dark matter with the real matter one can describe the wellknown pulsars (e.g. PSR J1748-2021B in NGC 6440B, PSR J1911-5958A in NGC 6752, PSR B1802-07 in NGC 6539 and PSR J1750-37A in NGC 6441 etc.) in a good manner in all respects. Therefore, we conclude that there is every possibility of existence of dark matter admixed with ordinary matter in the above mentioned pulsars.

Acknowledgements I acknowledge H. Haghi and A. Ghari of IASBS, Iran for providing the data of $v_{\text {halo }}^{2}$ which was calculated from the fitting of the rotation curves of the SPARC sample of galaxies. MK would like to thank IUCAA, Pune, India for providing research facilities and warm hospitality under Visiting Associateship where a part of this work was carried out.

\section{References}

1. S.L. Shapiro, S.A. Teukolsky, Black Holes, White Dwarfs and Neutron Stars: The Physics of Compact Objects (Wiley, New York, 1983)

2. A. Drago, A. Lavagno, G. Pagliara, Phys. Rev. D 89, $043014(2014)$

3. P. Haensel, Z.L. Zdunik, R. Schaeffer, A \& A 160, 121 (1986)

4. D. Leahy, R. Ouyed, Mon. Not. R. Astron. Soc. 387, 1193 (2008)

5. C. Alcock, E. Farhi, A. Olinto, Astrophys. J. 310, 261 (1986)

6. E. Farhi, R.L. Jaffe, Phys. Rev. D 30, 2379 (1984)

7. S. Postnikov, M. Prakash, J.M. Lattimer, Phys. Rev. D 82, $024016(2010)$

8. M. Dey, I. Bombaci, J. Dey, S. Ray, B.C. Samanta, Phys. Lett. B 438, 123 (1998)

9. J.M. Lattimer, M. Prakash, Phys. Rep. 442, 109 (2007)

10. F. Özel, Nature 441, 1115 (2006)

11. F. Özel, T. Güver, D. Psaitis, Astrophys. J. 693, 1775 (2009)

12. F. Özel, D. Psaitis, Phys. Rev. D 80, 103003 (2009)

13. F. Özel, G. Baym, T. Güver, Phys. Rev. D 82, 101301 (2010) 

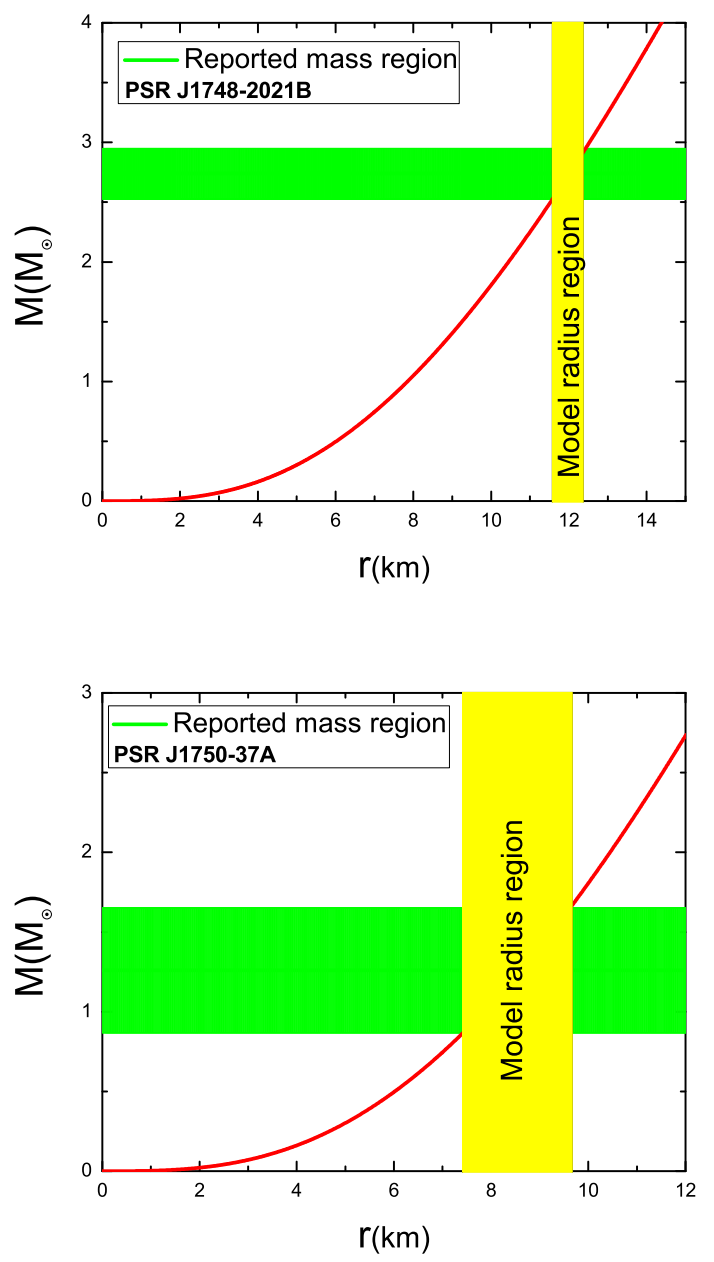

Fig. 8 Probable radii of Pulsars.

14. T. Güver, F. Özel, A. Cabrera-Lavers, Astrophys. J. 712, 964 (2010)

15. T. Güver, P. Wroblewski, L. Camarota, F. Özel, Astrophys. J. 719, 1807 (2010)

16. S.R. Heap, M.F. Corcoran, Astrophys. J. 387, 340 (1992)

17. J.M. Lattimer, M. Prakash, Phys. Rev. Lett. 94, 111101 (2005)

18. D. Stickland, C. Lloyd, A. Radzuin-Woodham, MNRAS 286, L21 (1997)

19. J.A. Orosz, E. Kuulkers, MNRAS 305, 132 (1999)

20. J.H. Van Kerkwijk, J. van Paradijis, E.J. Zuiderwijk, A\&A 303, 497 (1995)

21. F. Rahaman et al., Gen. Relativ. Gravit. 44, 107 (2012)

22. F. Rahaman et al., Eur. Phys. J. C 72, 2071 (2012)

23. M. Kalam et al., Eur. Phys. J. C 72, 2248 (2012)

24. M. Kalam et al., Int. J. Theor. Phys. 52, 3319 (2013)

25. M. Kalam et al., Eur. Phys. J. C 73, 2409 (2013)

26. M. Kalam et al., Eur. Phys. J. C 74, 2971 (2014)

27. M. Kalam et al., Astrophys. Space Sci. 349, 865 (2014)

28. M. Kalam et al., Mod. Phys. Lett. A 31, No. 40, 1650219 (2016)

29. M. Kalam et al., Mod. Phys. Lett. A 32, No. 4, 1750012 (2017)

30. M.A.K. Jafry et al., Astrophys. Space Sci. 362, no.10, $188(2017)$
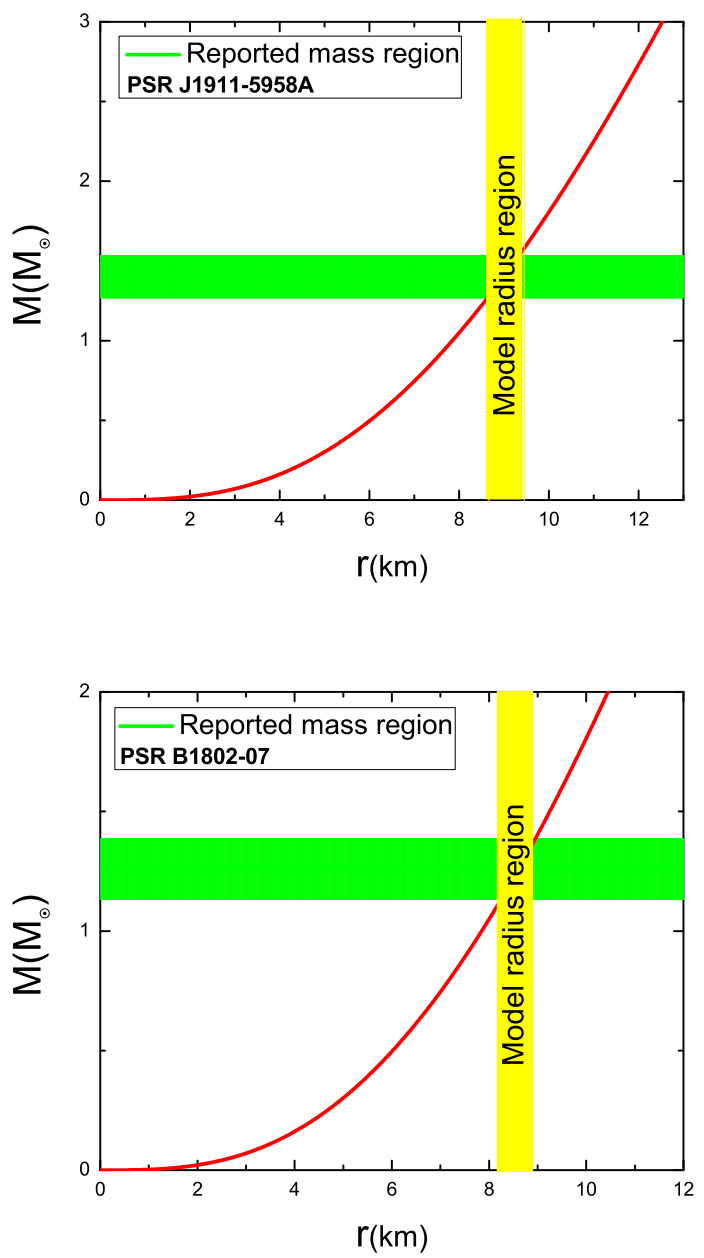

31. Sk.M. Hossein et al., Int. J. Mod. Phys. D 21, 1250088 (2012)

32. F. Lobo, Class. Quantum. Grav. 23, 1525 (2006)

33. K. Bronnikov and J.C. Fabris, Phys. Rev. Lett. 96, 251101 (2006).

34. S.K. Maurya, Y.K. Gupta, Baiju Dayanandan, Saibal Ray, Eur. Phys. J. C 76, 266 (2016)

35. B. Dayanandan, S.K. Maurya, Y.K. Gupta, T.T. Smitha, Astrophys Space Sci. 361, 160 (2016)

36. S.D. Maharaja, J.M. Sunzub, S. Ray, Eur. Phys. J. Plus 129, 3 (2014)

37. S. Ngubelanga, S.D. Maharaj, S. Ray, Astrophys Space Sci. 357, 74 (2015)

38. B.C. Paul, P.K. Chattopadhyay, S. Karmakar, Astrophys. Space Sci. 356, 327 (2015)

39. N. Pant, N. Pradhan, M.H. Murad, Int. J. Theor. Phys. 53, 11 (2014)

40. Piyali Bhar, Ksh. Newton Singh, N. Sarkar, F. Rahaman, Eur. Phys. J. C 77, 9 (2017)

41. F. Zwicky, Helv. Phys. Acta 6, 110 (1933); Gen. Relativ. Gravit. 41, 207 (2009).

42. V.C. Rubin, W.K. Ford, Jr., Astrophys. J. 159, 379 (1970)

43. K.A. Olive, arXiv:astro-ph/0301505

44. C. Munoz, Int. J. Mod. Phys. A 19, 3093 (2004) 
45. M. Taoso, G. Bertone, A. Masiero, J. Cosmol. Astropart. Phys. 03, 022 (2008)

46. I. Lopes, J. Silk, Astrophys. J. 722, L95 (2010)

47. C. Kouvaris, P. Tinyakov, Phys. Rev. D 82, 063531 (2010)

48. S. Turck-Chize, I. Lopes, Res. Astron. Astrophys. 12, 1107 (2012)

49. I. Lopes, J. Silk, Astrophys. J. 786, 25 (2014)

50. I. Lopes, K. Kadota, J. Silk, Astrophys. J. Lett. 780, 2 (2014)

51. R. Brito, V. Cardoso, H. Okawa, Phys. Rev. Lett. 115, 111301 (2015)

52. R. Brito, V. Cardoso, C.F.B. Macedo, H. Okawa, C. Palenzuela, Phys. Rev. D 93, 044045 (2016)

53. A. Martins, I. Lopes, J. Casanellas, Phys. Rev. D 95 , 023507 (2017)

54. A. Li, F. Huang, R.X. Xu, Astropart. Phys. 37, 70 (2012)

55. X. Li, F. Wang, K.S. Cheng, J. Cosmol. Astropart. Phys. 10, 031 (2012)

56. G. Panotopoulos, I. Lopes, Phys. Rev. D 96, 023002 (2017)

57. G. Narain, J. Schaffner-Bielich, I.N. Mishustin, Phys. Rev. D 74, 063003 (2006)

58. S.C. Leung, M.C. Chu, L.M. Lin, Phys. Rev. D 84, $107301(2011)$

59. S.C. Leung, M.C. Chu, L.M. Lin, Phys. Rev. D 85, 103528 (2012)

60. P. Mukhopadhyay, J. Schaffner-Bielich, Phys. Rev. D 93, 083009 (2016)

61. G. Panotopoulos, I. Lopes, Phys. Rev. D 96, 083004 (2017)

62. G. Panotopoulos, I. Lopes, Phys. Rev. D 96, 083013 (2017)

63. D.N. Spergel, P.J. Steinhardt, Phys. Rev. Lett. 84, 3760 (2000)

64. J. Ellis, A. Hektor, G. Hütsi, K. Kannike, L. Marzola, M. Raidal, V. Vaskonen, Phys. Lett. B 781, 607 (2018)

65. A. Nelson, S. Reddy, D. Zhou, arXiv:1803.03266

66. J. Ellis, G. Hütsi, K. Kannike, L. Marzola, M. Raidal, V. Vaskonen, Phys. Rev. D 97, 123007 (2018)

67. P.C.C. Freire et al., The Astrophysical Journal 675, 670 (2008)

68. C.G. Bassa et al., A \& A 456, 295 (2006)

69. S.E. Thorsett, D. Chakrabarty, The Astrophysical Journal 512, 288 (1999)

70. J.M. Lattimer, Annu. Rev. Nucl. Part. Sci. 62, 485-515 (2012)

71. C. Keeton " A catalog of Mass models for Gravitational Lensing", arXiv: astro-ph/0102341v2[astro-ph]

72. F. Sandin, P. Ciarcelluti, Astropart. Phys. 32, 278 (2009)

73. I. Goldman, R.N. Mohapatra, S. Nussinov, D. Rosenbaum, V. Teplitz, Phys. Lett. B 725, 200 (2013)

74. S.C. Leung, M.C. Chu, L.M. Lin, K.W. Wong, Phys. Rev. D 87, 123506 (2013)

75. S. Mukhopadhyay, D. Atta, K. Imam, D.N. Basu, C. Samanta, Eur. Phys. J. C 77, 440 (2017)

76. Z. Rezaei, Astrophys. J. 835, 33 (2017), arXiv:1612.02804 [astro-ph.HE]

77. Z. Rezaei, Int. J. Mod. Phys. D 27, 1950002 (2018), arXiv:1807.01781 [astro-ph.HE]

78. P.M. Takisa, S.D. Maharaj, L.L. Leeuw, Eur. Phys. J. C 79, 8 (2019)

79. D.K. Matondo, S.D. Maharaj, S. Ray, Eur. Phys. J. C 78, 437 (2018)

80. S.K. Maurya, Y.K. Gupta, S. Ray, S.R. Chowdhury, Eur. Phys. J. C 75, 389 (2015)
81. S.K. Maurya, A. Banerjee, M.K. Jasim, J. Kumar, A.K. Prasad, A. Pradhan, Phys. Rev. D 99, 044029 (2019)

82. B. Dayanandana, S.K. Maurya, Smitha T. T, Eur. Phys. J. A 53, 141 (2017)

83. S.K. Maurya, Eur. Phys. J. A 53, 89 (2017)

84. S. Gedela, R. K Bisht, N. Pant, Eur. Phys. J. A 54, 207 (2018)

85. K.N. Singh, N. Pant, M. Govender, Chinese Physics C 41, No. 1, 015103 (2017)

86. F. Rahaman, S.D. Maharaj, I.H. Sardar, K. Chakraborty, Mod. Phys. Lett. A 32, No. 8, 1750053 (15 pages) (2017)

87. M.K. Jasim, S.K. Maurya, Y.K. Gupta, B. Dayanandan, Astrophys Space Sci 361, 352 (2016)

88. P.M. Takisa, S.D. Maharaj, Astrophys Space Sci 361, $262(2016)$

89. S.K. Maurya, M.K. Jasim, Y.K. Gupta, T.T. Smitha, Astrophys Space Sci 361, 163 (2016)

90. K.N. Singh, N. Pradhan, N. Pant, Pramana - J. Phys. 89, $23(2017 \mathrm{a})$

91. H. Heintzmann, Z. Phys. 228, 489 (1969)

92. F. Brimioulle et al., Mon. Not. R. Astron. Soc. 432, 1046 (2013)

93. M. Oguri et al., Astrophys. J. 568, 488(2002)

94. J. Barranco et al., arXiv:1301.6785V1[astro-ph.CO]

95. F. Lelli, S. S. McGaugh, and J. M. Schombert, Astrophys. J. 152, 157(2016)

96. J.P. de León, Gen. Relativ. Grav. 25, 1123 (1993)

97. L. Herrera, Phys. Lett. A 165, 206 (1992)

98. H. Abreu, H. Hernandez, L.A. Nunez, Class. Quantum. Grav. 24, 4631 (2007)

99. S. Chandrasekhar, Astrophys. J. 140, 417 (1964)

100. J.M. Bardeen, K.S. Thorne, D.W. Meltzer, Astrophys. J. 145, 505 (1966)

101. H. Knutsen, MNRAS 232, 163 (1988)

102. M.K. Mak, T. Harko, Eur. Phys. J. C 73, 2585 (2013)

103. H.A. Buchdahl, Phys. Rev. 116, 1027 (1959)

104. P. Haensel, J.P. Lasopa, J.L. Zdunik, Nucl. Phys. Proc. Suppl. 80, 1110 (2000) 


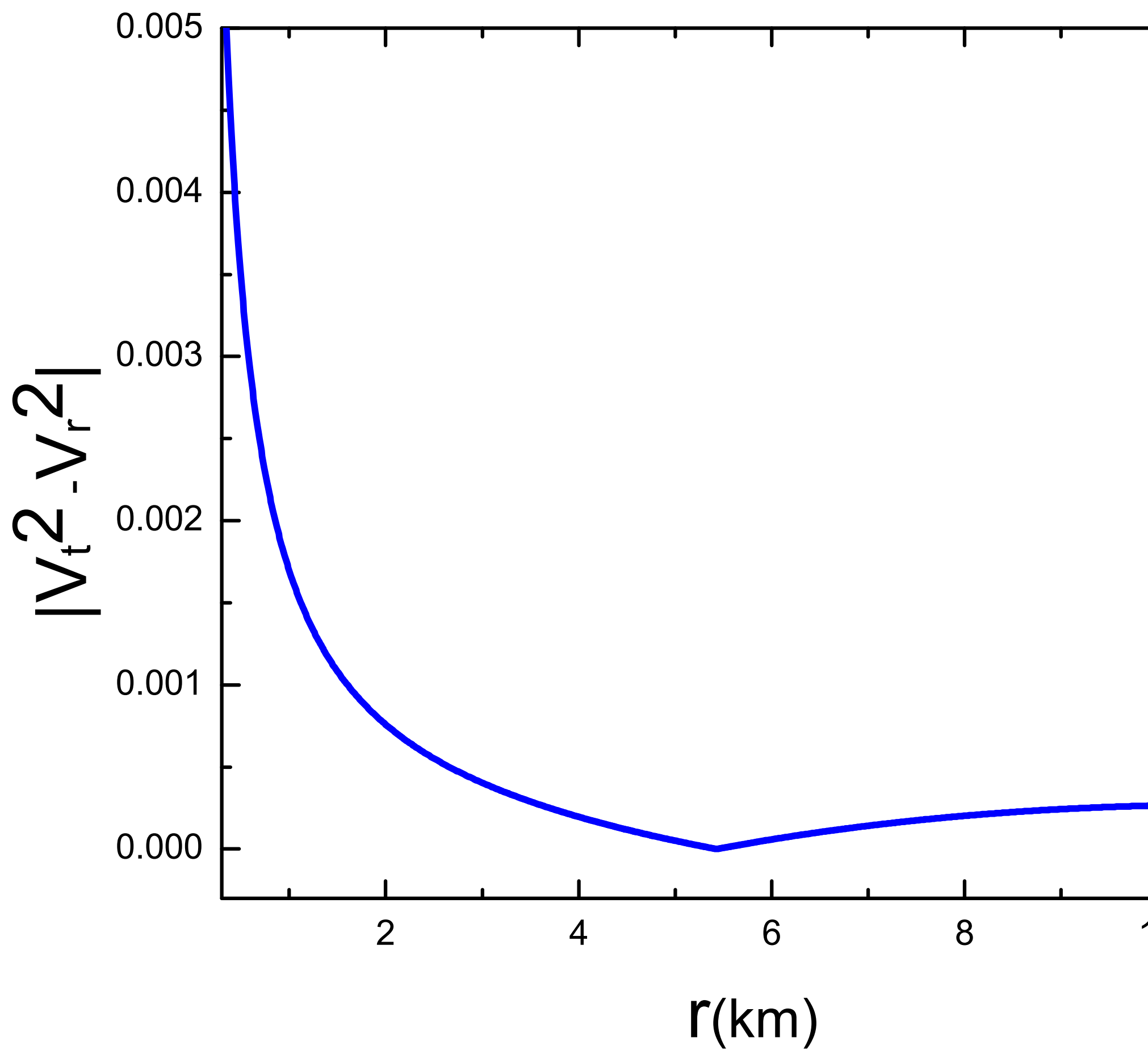

